\title{
Adolescentes tem fome de que? Fatores determinantes da escolha alimentar
}

\author{
Teens are hungry for what? Determinants of food choice \\ Adolescentes tienen hambre de qué? Determinantes de la elección de alimentos
}

Recebido: 30/06/2021 | Revisado: 07/07/2021 | Aceito: 09/07/2021 | Publicado: 19/07/2021

Kaio Germano Sousa da Silva

ORCID: https://orcid.org/0000-0003-4236-6230

Faculdade do Vale Elvira Dayrell, Brasil E-mail: kaiogsds@hotmail.com

Karine Costa Melo

ORCID: https://orcid.org/0000-0001-8253-859X

Universidade Federal do Maranhão, Brasil

E-mail: karinemelo09@gmail.com

Natália Monteiro Pessoa

ORCID: https://orcid.org/0000-0001-6502-5661

Instituto de Pesquisas, Ensino e Gestão em Saúde, Brasil

E-mail: nataliatulip@ hotmail.com

Rondinelle dos Santos Chaves

ORCID: https://orcid.org/0000-0003-4941-8005

Instituo Sírio-Libanês de Ensino e Pesquisa, Brasil

E-mail: rondinelledsch@ hotmail.com

Raphaela Lau da Silva e Silva

ORCID: https://orcid.org/0000-0002-3525-6394

Universitário de Ciências e Tecnologia do Maranhão, Brasil

E-mail: mwashingtonwalber@gmail.com

Luana Pereira Ibiapina Coêlho

ORCID: https://orcid.org/0000-0002-2054-959X

Faculdade de Venda Nova do Imigrante, Brasil

E-mail: luanaibiapina@hotmail.com

Eduardo Brito da Silva

ORCID: https://orcid.org/0000-0002-8571-7806

Centro Universitário de Ciências e Tecnologia do Maranhão, Brasil

E-mail: eduzinhobds@gmail.com

Washington Walber Macedo dos Santos

ORCID: https://orcid.org/0000-0003-4577-1143

Centro Universitário de Ciências e Tecnologia do Maranhão, Brasil

E-mail: mwashingtonwalber@gmail.com

Andréa Gomes de Almeida

ORCID: https://orcid.org/0000-0002-4221-0562

Centro Universitário de Ciências e Tecnologia do Maranhão, Brasil

E-mail: almeidaandrea709@gmail.com

Valéria Bandeira Leal

ORCID: https://orcid.org/0000-0002-4024-702X

Centro Universitário de Ciências e Tecnologia do Maranhão, Brasil

E-mail: valerialeaw@ hotmail.com

Bruna Rafaelle Pereira Ibiapina Coêlho

ORCID: https://orcid.org/0000-0002-3122-4803

Escola de Saúde Pública do Ceará, Brasil

E-mail: bruna.ibiapina@ hotmail.com

Karolayne Brito Silva

ORCID: https://orcid.org/0000-0002-3679-9091

Centro Universitário de Ciências e Tecnologia do Maranhão, Brasil

E-mail: karolaynebrito2018@outlook.com

Ana Carla Marques da Costa

ORCID: https://orcid.org/0000-0002-4246-145X Universidade Luterana do Brasil, Brasil

E-mail: carla_ma27@hotmail.com

Eudilene da Silva Mesquita

ORCID: https://orcid.org/0000-0001-9894-0903

Centro Universitário de Ciências e Tecnologia do Maranhão, Brasil

E-mail: eu.mesquitta@gmail.com

Pedro Henrique Medeiros de Andrade

ORCID: https://orcid.org/0000-0003-1819-9540

Centro Universitário de Ciências e Tecnologia do Maranhão, Brasil E-mail: pedro.medeiroscx@gmail.com 


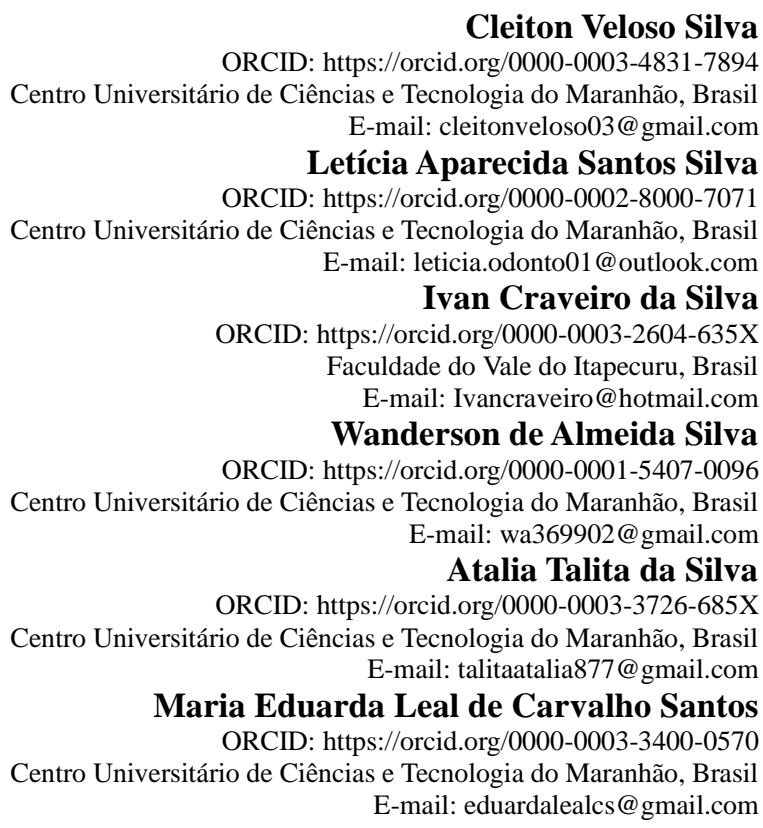

Cleiton Veloso Silva

ORCID: https://orcid.org/0000-0003-4831-7894 Centro Universitário de Ciências e Tecnologia do Maranhão, Brasil E-mail: cleitonveloso03@gmail.com

Letícia Aparecida Santos Silva ORCID: https://orcid.org/0000-0002-8000-7071 Centro Universitário de Ciências e Tecnologia do Maranhão, Brasil E-mail: leticia.odonto01@ outlook.com

Ivan Craveiro da Silva

ORCID: https://orcid.org/0000-0003-2604-635X Faculdade do Vale do Itapecuru, Brasil E-mail: Ivancraveiro@ hotmail.com

Wanderson de Almeida Silva

ORCID: https://orcid.org/0000-0001-5407-0096 Centro Universitário de Ciências e Tecnologia do Maranhão, Brasil E-mail:wa369902@gmail.com

Atalia Talita da Silva

ORCID: https://orcid.org/0000-0003-3726-685X Centro Universitário de Ciências e Tecnologia do Maranhão, Brasil E-mail: talitaatalia877@gmail.com

Maria Eduarda Leal de Carvalho Santos

ORCID: https://orcid.org/0000-0003-3400-0570 Centro Universitário de Ciências e Tecnologia do Maranhão, Brasil E-mail: eduardalealcs@gmail.com

\title{
Resumo
}

Adolescência é definida cronologicamente pela a Organização Mundial da Saúde (OMS) para caracterizar indivíduos que tem entre 10 e 19 anos, este estudo objetivou realizar uma revisão narrativa sobre o perfil alimentar de adolescentes. Trata-se de uma pesquisa qualitativa, utilizando uma análise bibliográfica de caráter narrativo realizada através de consulta em bases de dados online de pesquisa: Scielo (Scientific Eletronic Library OnLine), BVS (Biblioteca Virtual da Saúde), e Google acadêmico, onde pesquisa foi realizada com o recorte temporal de 2016 a 2021. Como resultado constatou-se que a alimentação dos adolescentes é em sua maioria é composta por gordura, sódio e açúcares simples, que estão acompanhados do sedentarismo. A alimentação dos adolescentes é composta basicamente por alimentos ricos em açucares e elevado teor de gordura, baixo consumo de frutas e hortaliças o que favorece o ganho de peso acima do ideal e maior probabilidade de desenvolvimento de Doenças Crônicas Não Transmissíveis. Conclui-se neste aspecto, os adolescentes têm consumido principalmente produtos industrializados processados e ultraprocessados, diminuindo a saúde física e psicologia e aumentando o problema da obesidade em todo o mundo. Esses produtos têm preços acessíveis e são amplamente divulgados na mídia, passando a falsa sensação de que se pode consumi-los à vontade sem ocasionar malefícios à saúde.

Palavras-chave: Adolescente; Nutrição do adolescente; Comportamento de doença.

\begin{abstract}
Adolescence is defined chronologically by the World Health Organization (WHO) to characterize individuals who are between 10 and 19 years old. This study aimed to carry out a narrative review on the dietary profile of adolescents. This is a qualitative research, using a bibliographic analysis of a narrative character carried out by consulting online research databases: Scielo (Scientific Electronic Library OnLine), BVS (Virtual Health Library), and Academic Google, where the research was carried out with the time frame from 2016 to 2021. As a result, it was found that the diet of adolescents is mostly composed of fat, sodium and simple sugars, which are accompanied by a sedentary lifestyle. Adolescents' diet is basically composed of foods rich in sugar and high in fat, low consumption of fruits and vegetables, which favors weight gain above the ideal and greater likelihood of developing Chronic NonCommunicable Diseases. It is concluded in this aspect, adolescents have mainly consumed processed and ultraprocessed industrialized products, decreasing their physical health and psychology and increasing the problem of obesity worldwide. These products have affordable prices and are widely publicized in the media, giving the false feeling that they can be consumed freely without causing harm to health.
\end{abstract}

Keywords: Adolescent; Adolescent nutrition; Illness behavior.

\section{Resumen}

La adolescencia es definida cronológicamente por la Organización Mundial de la Salud (OMS) para caracterizar a las personas de entre 10 y 19 años, este estudio tuvo como objetivo realizar una revisión narrativa sobre el perfil dietético de los adolescentes. Se trata de una investigación cualitativa, mediante un análisis bibliográfico de carácter narrativo realizado mediante la consulta de bases de datos de investigación en línea: Scielo (Scientific Electronic Library OnLine), BVS (Virtual Health Library) y Academic Google, donde se realizó la investigación con el tiempo. marco de 2016 a 2021. Como resultado, se encontró que la dieta de los adolescentes está compuesta mayoritariamente por grasas, sodio y azúcares simples, los cuales van acompañados de un estilo de vida sedentario. La dieta de los 
adolescentes está compuesta básicamente por alimentos ricos en azúcar y altos en grasas, bajo consumo de frutas y verduras, lo que favorece el aumento de peso por encima de lo ideal y una mayor probabilidad de desarrollar Enfermedades Crónicas No Transmisibles. Se concluye en este aspecto, los adolescentes han consumido principalmente productos industrializados procesados y ultraprocesados, disminuyendo su salud física y psicológica y aumentando el problema de la obesidad a nivel mundial. Estos productos tienen precios asequibles y son ampliamente publicitados en los medios, dando la falsa sensación de que pueden consumirse libremente sin causar daños a la salud.

Palabras clave: Adolescente; Nutrición del adolescente; Conducta de enfermedad.

\section{Introdução}

Adolescência é definida cronologicamente pela a Organização Mundial da Saúde (OMS) para caracterizar indivíduos que tem entre 10 e 19 anos. Nesta fase de crescimento e desenvolvimento, existe vários fatores que pode referenciar ou influenciar seus hábitos alimentares podendo causar uma escolha errônea do alimento (WHO, 2014).

Diante dessas alterações, os adolescentes demonstram diversas preocupações, entre elas a aparência corporal, que pode desencadear alterações no seu comportamento alimentar, sob influência de fatores internos, como a autopercepção corporal, necessidades fisiológicas, saúde individual e desenvolvimento psicossocial, além dos fatores externos, como hábitos familiares, amizades, modismo e regras sociais (Engstrom \& Barros, 2019).

Neste raciocínio os determinantes dos hábitos e o conhecimento sobre a alimentação tem uma repercussão sobre o comportamento e estilo de vida futuramente, como a autoimagem influenciada pela mídia, saúde pessoal, valores, referências e desenvolvimento psicossocial. Onde hábitos alimentares errados em adolescentes podem ser fatores determinantes de risco para o desenvolvimento de doenças crônicas na fase adulta, destacando-se a importância da alimentação no período de pandemia atual, onde diversos alimentos apresentam propriedades nutracêuticas e podem aumentar a imunidade (Levy et al., 2017; Soares et al., 2021).

Deste modo o consumo de alimentos com baixo teor de gorduras e açucares, ricos em vitaminas, minerais e fibras, correlacionadas a atividade física tem um papel muito importante no crescimento e no desenvolvimento dos adolescentes, com foco principal no combate a obesidade, uma patologia de causa multifatorial. Estudos tem demostrado evidencias que adolescentes com excesso de peso (sobrepeso e obesidade) geralmente tem uma maior ingestão energética e atividade física insatisfatória (Pinho \& Martínez, 2014).

No Brasil, déficit de altura, problema que acomete os adolescentes, teve diminuição em mais de três vezes desde 1974, porem a prevalência de obesidade em adolescentes vem aumentado cada vez mais expressando um aspecto negativo neste processo. A incidência de excesso de peso vem aumentando muito no decorrer dos anos, sendo que cresceu mais de quatro vezes entre os meninos e duas vezes entre as meninas no mesmo período (Taddei, Lang, Longo-Silva, \& Toloni, 2021).

Muitos estudos vêm mostrando, problemas com relação principalmente na escolha alimentar correta preferindo alimentação pouco saudável, tendo preferências por dietas usualmente com um alto teor em gorduras, açúcares e sódio, sendo que esses compostos são ingredientes muito utilizados e encontrados em grandes quantidades em alimentos industrializados. $\mathrm{O}$ comportamento alimentar este ligado a padrões de estilo de vida adotado pelo grupo etário ao qual pertence, como a omissão de refeições (Silva et al., 2021).

Em adolescentes, pode-se considerar que o sobrepeso é a alteração nutricional em ascensão, em que a prevalência de excesso de peso em crianças e adolescentes nos países em desenvolvimento é de 12,9\% em meninos e 13,4\% em meninas. Ademais, nos países desenvolvidos, esses valores são ainda maiores, onde 23,8\% dos meninos e $22,6 \%$ das meninas apresentam sobrepeso ou obesidade (D'avila, 2018).

Com isso o estudo teve como problemática “Quais fatores determinam o perfil alimentar dos adolescentes?” E como objetivo realizar uma revisão narrativa sobre o perfil alimentar de adolescentes. Justifica-se o estudo dos hábitos alimentares deste grupo, pois o Brasil tem apresentado profundas modificações no perfil nutricional de sua população, fruto de um 
processo conhecido como transição nutricional, havendo uma diminuição na desnutrição e aumento da obesidade, em intensidades diferentes, dependendo da região e do estrato social.

\section{Metodologia}

Estudos de revisão são divididos em narrativos, integrativos ou sistemáticos, trazendo para este trabalho, o mesmo consistiu-se numa pesquisa qualitativa (Potter \& Perry, 2013) utilizando uma análise bibliográfica de caráter narrativo realizada através de consulta em bases de dados online de pesquisa: Scielo (Scientific Eletronic Library OnLine), BVS (Biblioteca Virtual da Saúde), e Google acadêmico (Silva et al., 2021). A pesquisa foi realizada com o recorte temporal de 2016 a 2021.

Foram utilizados os seguintes descritores e suas consonâncias nas línguas portuguesa, espanhola e inglesa: "Adolescência 1", "Consumo Alimentar 2", "Comportamento Alimentar 3". Vocábulos com grau de semelhança foram usados em outras bases de dados. Seguindo o trajeto metodológico, foi identificado 36 artigos de acordo com esses termos usados. Em seguida, fez-se a leitura detalhada, com resultado de 26 artigos, sendo 6 artigos oriundos do Google acadêmico, 10 oriundos da Scielo e 10 oriundos da BVS.

No que se refere aos critérios de inclusão: Identificação e caracterização do artigo, ano de publicação no recorte temporal (2016 a 2021) e clássicos, artigos fora do recorte temporal, mas de grande relevância, e que tratavam do tema proposta. Também houve pesquisa em documentos governamentais e foram incluídos os trabalhos que relacionaram com a temática.

Quanto aos critérios de exclusão, foram excluídos artigos com formato de cartas ao editor, comentários, casos em séries, resumos que não foram publicados, trabalhos que não se adequavam com os objetivos e ao tema proposto em conjunto com os descritores "1","2", “3”; aqueles que não se enquadravam no recorte temporal supracitados acima, e os que não se apresentavam disponíveis em textos completos. Os artigos foram lidos de forma minuciosa e crítica, e foram selecionados os textos que melhor abordaram o assunto.

\section{Resultados e Discussão}

\section{Adolescência e Consumo Alimentar}

A Organização Mundial da Saúde (OMS) determina que a adolescência compreende o período da vida entre os 10 a 19 anos de idade. Esta etapa da vida é marcada por um processo de intensas modificações no crescimento e de desenvolvimento biológico, psicológico, físico e social do indivíduo (Nery, Mendonça, Gomes, Fernandes, \& Oliveira, 2017).

Conforme Chaves (2018) durante a fase da adolescência ocorre um rápido crescimento das estruturas corporais e consequentemente das necessidades energéticas e de nutrientes com intuito de garantir essas modificações, portanto uma dieta balanceada é capaz de permitir a expressão total dos genes relacionados ao desenvolvimento do corpo.

A alimentação de adolescentes necessita de um enfoque especial devido a fase de crescimento e desenvolvimento que requer uma dieta balanceada, a fim de atender todas as necessidades fisiológicas e metabólicas (Gomes, Schmidt, \& Biesek, 2017).

$\mathrm{Na}$ adolescência, a alimentação saudável é essencial para garantir o desenvolvimento físico e mental, o crescimento e maturação sexual além de prevenir futuramente contra possíveis doenças (Vieira, Del Ciampo, \& Del Ciampo, 2018). Segundo Tavares, Yakoo, Rosa e Fonseca (2017) os adolescentes são um dos grupos mais vulnerável a ingestão alimentar inapropriada e estado nutricional inadequado, devido aos hábitos alimentares incorretos e as modificações biológicas que ocorrem nessa fase da vida.

No que diz respeito à alimentação dos adolescentes, pode-se observar que em sua maioria é composta por gordura, 
sódio e açúcares simples, que estão acompanhados do sedentarismo (Pereira, Pereira, \& Angelis-Pereira, 2017). A alimentação dos adolescentes é composta basicamente por alimentos ricos em açucares e elevado teor de gordura, baixo consumo de frutas e hortaliças o que favorece o ganho de peso acima do ideal e maior probabilidade de desenvolvimento de Doenças Crônicas Não Transmissíveis (Enes \& Slater, 2019).

Alguns estudos disponíveis na literatura apontam que os adolescentes de classe econômica mais favorecida e com maior acesso a informações apresentam hábitos alimentares incorreto em relação a escolha dos alimentos, preferindo em sua maioria alimentos com alto teor de carboidratos e sódio e baixa ingestão de vegetais (Toral, Conti, \& Slater, 2019).

Estudos mostram que há uma alteração dos principais alimentos ingeridos pelo brasileiro, caracterizando-se pela diminuição do consumo de arroz e feijão, principalmente por adolescente, e aumento do consumo de fast foods e doces, o que consequentemente ocasiona o aumento do número de pessoas com obesidade, hipertensão arterial, síndromes metabólicas, dentre outras patologias (Salvatti, Escrivão, Taddei, \& Bracco, 2017).

Entretanto é importante destacar que a maioria dos jovens apresenta uma preocupação excessiva, alguns para emagrecer e outro para ganhar peso, o que traz como consequência o desenvolvimento de transtornos alimentares como anorexia nervosa, bulimia nervosa, e transtorno alimentar não especificado (Veiga, 2018).

Enfim, os adolescentes ao realizar uma refeição buscam um contentamento imediato a fim de satisfazer os desejos, porém não se preocupam com as consequências futuras de uma alimentação não equilibrada (Bica, 2018). A adoção de um comportamento alimentar saudável contribui para adequação do peso ideal, contudo para realização desta prática é necessário conhecimento sobre nutrição e alimentação (Rezende, 2020).

\section{Determinantes Alimentares Relacionados a Mídia}

A mídia é considerada o meio de comunicação com maior capacidade de influência sobre os adolescentes, não só pelos seus objetos de influência como televisão, redes sociais, internet, radio e outros meios de divulgação como revistas que são bem populares entre esses, como também pelo tempo que os jovens ficam expostos a essas mídias. Pesquisas têm apontado um aumento perceptível do tempo gasto com o hábito de assistir TV (Reato, 2017).

Nos Estados Unidos, adolescentes assistem em média 22h de televisão por semana, sendo o tempo gasto com a TV muitas vezes maior do que o tempo utilizado com qualquer outra atividade de lazer (Silva et al., 2021). No Brasil, estudo realizado por Silva e Malina (2018) aponta para dados ainda mais preocupantes - cerca de 4,4 a 4,9 horas por dia são gastas pelos adolescentes diante da televisão, um valor bem acima da média americana.

Pesquisas apontam que existe uma relação do hábito de comer enquanto se assiste televisão, com uma alimentação não saudáveis e com excesso de peso, ou seja, comer em frente à televisão, celulares e computadores ou em frente de qualquer outra coisa que desvie a atenção da alimentação, faz com que esses indivíduos tenham formação de hábitos alimentares não saudáveis (Levy et al., 2020).

Segundo Silva et al. (2021) a propaganda tem sua parcela de influência, já que grande empresa no ramo de alimentação investe milhões em divulgar seus produtos que usa de vários fatores nessas propagandas como o estímulo do objeto de comer, a ilusão associada com fantasia, a figura da família e amizade, curiosidade e o sabor do alimento juntamente com outros meios como músicas ou o prazer que o alimento causa

O apelo nutricional, preço e conscientização são temas menos discutidos nos comerciais, dessa forma percebe-se que os recursos emocionais são utilizados de forma estratégicos altamente utilizados para sensibilizar o público, público esse formado por adolescentes ou pais também (Milani, Garlet, Romero, \& Mattos, 2017). 
Para adolescentes, prevaleceram os comerciais de achocolatados, balas, cereais, chocolates e refrigerantes, com utilização de temas relacionados a essa fase como o "ficar" e "curtir" ambientados em lugares preferidos desse público, como praças, colégios e atualmente usando a internet (Silva et al., 2019).

Essa divulgação apela principalmente para o desejo dos jovens de conquistar parceiros e se aventurarem. Nota-se que as propagandas de alimentos em geral enfatizam as emoções em suas mensagens, em sua maioria associando o consumo do produto a uma sensação de prazer, com recompensas sociais e satisfação do ego (Santos, 2019). Considerações sobre ingredientes e textura dos alimentos normalmente não possuem caráter informativo e sim persuasivo, apelando para o apetite do consumidor (Santos, 2017).

Além disso, a mídia molda comportamentos através de exemplos normalmente pessoas bonitas e bem sucedidas, extremamente magras, anunciando refrigerantes, redes de fast food e outros alimentos, via de regra ricos em açúcar e gordura. Isso acaba influenciando negativamente os valores dos jovens, que passam a achar o consumo desses alimentos normal ou pertinente, e criam expectativas irreais sobre as consequências de maus hábitos alimentares, já que as estrelas da televisão raramente são obesas ou sofrem de problemas de saúde relacionados à má alimentação. Ao contrário dessas "estrelas", as crianças e os adolescentes estão se tornando cada vez mais obesos, apresentando risco de desenvolver diversas doenças crônico-degenerativas. (Oliveira, Rodeghiero, Peter, \& Muniz, 2021).

Além do incentivo ao consumo de determinados alimentos, o tempo gasto vendo televisão limita a realização de atividades importantes, como brincar, ler, praticar atividades físicas e interagir com amigos e familiares, o que contribui para a inatividade, cultivando um hábito sedentário que leva a uma diminuição do gasto energético, sendo assim um importante fator associado à obesidade e ao desenvolvimento de doenças crônicas (Silva \& Malina, 2018).

\section{Determinantes Alimentares Familiar}

Para uma vida saudável, e hábitos alimentares corretos do ponto de vista nutricional, destaca-se a promoção da saúde por meio de movimentos que visam motivar o indivíduo a adotar hábitos saudáveis de educação alimentar (Ferreira, Farias, \& Silvares, 2010).

O desenvolvimento e crescimentos do indivíduo tem seus momentos de expressividade desde a infância a adolescência. Nesse período ocorrem modificações nos hábitos de vida, que se concretizam e persistem ao longo de toda a vida. Dentre estes hábitos desenvolvidos, podemos enfatizar a alimentação, tendo uma grande influência no seu estado nutricional, pois a inadequação pode acarretar em problemas no desenvolvimento e até mesmo acarretar no desenvolvimento de futuras patologias, tanto no que diz respeito ao excesso quanto à restrição alimentar (Milani, Garlet, Romero, \& Mattos, 2017).

Portanto o comportamento alimentar é um fator de risco para o desenvolvimento de uma série de patologias, ocupando um papel fundamental na prevenção, controle e tratamento de doenças. A alimentação durante as fases da vida, ao mesmo tempo em que tem sua importância no desenvolvimento, pode ser o principal fator, ligada a prevenção de algumas doenças que podem ser desenvolvidas ao decorrer do tempo. Cotidianamente é notório que a família, os fatores sociais e os ambientais influenciam diretamente o padrão alimentar desses indivíduos (Cornwell et al., 2018).

Contudo a família aqui pode está diretamente ligada a estas etapas, sendo vista como um contexto nuclear no qual os hábitos de vida, ações e comportamentos sofrem influência. Dessa forma, a família de uma forma geral, principalmente os mais próximos, influencia fortemente no estado de saúde de cada um, assim como também o modo pelo qual esta unidade funcionara. Essa influência reciproca pode ser por laços biológicos, legais ou reais (Cediel et al., 2018)

Enfim a família tem influência direta na saúde e desenvolvimento do hábito das crianças e adolescentes é importante que estas estabeleçam e possuam conhecimentos para a adequação alimentar dos seus filhos (Pinto, 2017). Porém no que diz 
respeito ao consumo alimentar, visualiza-se um padrão inadequado desta faixa etária, evidenciando consumos exacerbados de alimentos ricos em gorduras e açúcares simples, baixa ingestão de frutas, verduras e legumes (Louzada et al., 2018).

\section{Hábitos Alimentares no Âmbito Escolar}

Visto que a adolescência se caracteriza por um período de intensas modificações que sofrem influência pelos hábitos familiares, condições socioeconômicas, amizades, valores e regras sociais e culturais, assim como também a experiência e conhecimento do próprio indivíduo, que irão repercutir em muitos aspectos em sua vida adulta, onde esses hábitos respectivamente são alimentares, autoimagem, saúde individual, preferência, valores e desenvolvimentos psicossociais (Levy et al., 2017).

Por tanto a escola é um ambiente capaz de interagir e integrar vários segmentos sociais através de metodologias que seguem desde o construtivismo ao sóciointeracionismo (Azevedo, Santos, Azevedo, \& Santos 2017), pois o ambiente escolar, por si só é considerado um cenário de oportunidades para expandir o acesso a uma alimentação saudável com estratégias de intervenções nutricionais (Araújo, Ferreira, Neumann, Miranda, \& Pires, 2017).

Por isso na vida adulta as características dos hábitos alimentares são absorvidas e/ou concretizadas na infância e na adolescência. O ambiente escolar é onde estes passam a maior parte do seu dia, onde são influenciados pelo meio, sendo relevante a realização de educação nutricional, consistindo em uma estratégia para contrapor as alimentações prejudiciais, proporcionando o conhecimento e a incorporação de novas expectativas que influenciam em seu padrão alimentar ao decorrer da vida (Avelar, Soares, Ferreira, 2017).

Por fim o a escola é uma instituição que apresenta grande potencial na realização das ações educativas, principalmente da educação alimentar e nutricional para que haja uma prevenção e controle no desenvolvimento de doenças. A respeito disto, o potencial da escola na formação dos hábitos alimentares é imenso. Pois ela não está envolvida em um simples momento de alimentação cotidiano dos alunos, mas esta é responsável por uma parcela considerável do conteúdo informativo adquirido e absorvido, inclusive no que diz respeito da educação alimentar e nutricional (Araújo et al., 2017).

Por isso nas refeições oferecidas nas escolas, é importante que elas sejam compostas por uma porção fonte de carboidrato, uma de fonte de proteína e uma de fruta, para fornecer a ingestão de fibras, minerais e vitaminas, além de uma de bebida, preferencialmente sem adição de açúcar, o que ira garantir a hidratação. Isso para que haja uma educação alimentar e seja armazenada pelos adolescentes e levada ao longo da vida (Fisberg, Previdelli, Del'arco, Tosatti, \& Nogueira, 2017).

\section{Doenças Relacionadas à Alimentação Inadequada na Adolescência (DRAIA)}

Destaca-se a obesidade tem causa multifatorial relacionada a um conjunto de influencias como os fatores biológicos, comportamentais e ambientais, contudo, entre muitos fatores que podem acometer a obesidade existe uma relação que se destaca, mas que são os hábitos alimentares inadequados e o sedentarismo. Essa doença tem como principal característica o acúmulo excessivo de gordura corporal capaz de trazer prejuízos à saúde, tais como hipertensão arterial, cardiopatias, diabetes entre outras (Silva, Magalhaes, Borba \& Sousa, 2019). E a hipertensão arterial sistêmica que está ligada a obesidade (Silva et al., 2021).

Destaca-se ainda, os transtornos alimentares que são doenças relacionadas ao fomento rápido de peso, tais doenças utiliza de estratégias não saudáveis para conseguir tais resultados, esses transtornos alimentares são induzidos de forma devastadora pela a mídia e a sociedade (Silva et al., 2019).

Anorexia nervosa é uma doença psiquiátrica relacionada a um transtorno alimentar no qual a pessoa, pode esta visivelmente saudável, vai à busca de estratégias para perda de peso rápido importante emagrecimento, tal transtorno pode ser fatal levando a morte por desnutrição. Essa doença ocorre principalmente em mulheres adolescentes e adultas jovens (12 a 20 
anos). A pessoa com essa doença vê imagens do seu corpo distorcidas, sempre com peso maior do que realmente possui, e para a perda de peso a pessoa utiliza estratégias como o uso de laxantes e diuréticos, jejuns, dietas restritas, exercícios físicos intensos e vômitos induzidos que podem levar a bulimia (Araguaia, 2017).

A bulimia nervosa consiste na ingestão descontrolada de alimentos, seguida de métodos para expulsão dos alimentos ingeridos, tais como autoindução de vômitos, uso indiscriminado de laxantes, diuréticos e prática excessiva de exercícios físicos (Cruz \& Coelho, 2018).

\section{Considerações Finais}

Este estudo permitiu observar os hábitos alimentares dos adolescentes atualmente e os fatores externos e internos que influenciam suas escolhas. Foi evidenciado que os jovens passam por diversas transformações durante a adolescência e os grupos sociais que frequentam podem induzir certas escolhas alimentares, vale destacar ainda, o papel das mídias sociais, pois os jovens são expostos aos seus conteúdos diariamente por longos períodos de tempo.

Neste aspecto, os adolescentes têm consumido principalmente produtos industrializados processados e ultraprocessados, diminuindo a saúde física e psicológica e aumentando o problema da obesidade em todo o mundo. Esses produtos têm preços acessíveis e são amplamente divulgados na mídia, passando a falsa sensação de que se pode consumi-los à vontade sem ocasionar malefícios à saúde.

Por fim, o estudo possibilitou expandir os conhecimentos sobre transtornos alimentares, obesidade, o perfil alimentar dos adolescentes e os fatores que influenciam suas escolhas. Dessa forma, considera-se que novas pesquisas devem ser realizadas nesta linha de investigação, a fim de provar a importância da alimentação saudável na adolescência e evidenciar seus benefícios físicos e psicológicos.

\section{Referências}

Araguaia, M. (2017). Anorexia. Distúrbio alimentar que pode causar diversos problemas fisiológicos e psíquicos. Brasil Escola.

Araújo, A. L., Ferreira, V. A., Neumann D., Miranda, L. S., \& Pires I. S. C. (2017). O impacto da educação alimentar e nutricional na prevenção do excesso de peso em escolares: uma revisão bibliográfica. Rev. Brasileira de Obesidade, Nutrição e Emagrecimento, 11(62), $24-104$.

Avelar, P. S., Soares, R. C. S., \& Ferreira, L. M. (2017). Educação alimentar e nutricional na escola: A Associação de Pais e Amigos dos Excepcionais de Viçosa como promotora de hábitos alimentares saudáveis. Rev. J Manag Prim Heal Care. 7(1), 125-125.

Azevedo, D. O., Santos, J. R., Azevedo, D. O., \& Santos, D. B. (2017). Implantação de novos espaços de aprendizagem na formação de crianças do ensino fundamental II: um estudo de caso na escola Visconde de Cairu, Salvador - Bahia. Rev.Cadernos Macambira, 1(2), 19-25.

Bica, I., Cunha, M., Costa, J., Rodrigues, V., Santos, M., \& Montero, J. (2018). Hábitos alimentares na adolescência: implicações no estado de saúde. Millenium.

Cornwell, B., Villamor, E., Mora-Plazas, M., Marin, C., Monteiro, C. A., \& Baylin, A. (2018). Processed and ultra-processed foods are associated with lowerquality nutrient profiles in children from Colombia. Public Health Nutr, 21(1), 142-147.

Cediel, G., Reyes, M., Louzada, M. L. C., Steele, E. M., Monteiro, C. A., Corvalán, C., \& Uauy, R. (2018). Ultra-processed foods and added sugars in the Chilean diet. Public Health Nutr, 21(1), 125-133.

Chaves, O. C. (2018). Influência do estado nutricional dos pais sobre o estado nutricional do filho adolescente, do município de Viçosa -MG em 2008. Dissertação (Mestrado em Ciência da Nutrição) - Departamento de Nutrição e Saúde, Universidade Federal de Viçosa, Viçosa.

Cruz, L. B., \& Coelho, R. S. (2018). Increasing consumption of ultra-processed foods and likely impact on human health: evidence from Brazil. Public Health Nutr, 14(1), 5-13.

D'avilah, K. V. (2017). Consumo energético proveniente de alimentos ultraprocessados por adolescentes. Rev Paul Pediatr, 35(1), 54-60.

Enes, C. C., \& Slater, B. (2019). Variation in dietary intake and physical activity pattern as predictors of change in body mass índex (BMI) Z-score among Brazilian adolecents. Revista Brasileira de Epidemiologia, 16(2), 493-501.

Engstrom, E. M., \& Barros, D. C. (2019). Diagnóstico nutricional de adolescentes. Sisvan: instrumento para o combate aos distúrbios nutricionais em serviços de saúde: o diagnótico nutricional. Fiocruz, 105-23. 
Ferreira, T. H. S., Farias, M. A., \& Silvares, E. F. D. M. (2010). Adolescência Através dos Séculos. Psicologia: Teoria e Pesquisa, 26(2), $227-234$.

Fisberg, M., Previdelli A. N., Del'arco, A. P. W. T., Tosatti, A., \& Nogueira, D. A. C. A. (2017). Hábito alimentar nos lanches intermediários de crianças escolares brasileiras de 7 a 11 anos: estudo em amostra nacional representativa. Rev. International Journal of Nutrology, 9(4), 225-236.

Gomes, J. S., Schmidt, M., \& Biesek, S. (2017). Avaliação do perfil antropométrico e consumo alimentar adolescentes jogadores de futsal. Revista Brasileira de Obesidade, Nutrição e Emagracimento. 9(53), 463-470.

Levy, R. B., Castro, I. R. R., Cardoso, L. O., Tavares, L. F., Sardinha, L. M. V., Gomes, F. S., Costa, A. W. C. (2017). Consumo e comportamento alimentar entre adolescentes brasileiros: Pesquisa Nacional de Saúde do Escolar (PeNSE), 2009. Rev.Ciência \& Saúde Coletiva, 15(2).

Levy, R. B., Castro, I. R. R., Cardoso, L. F., Sardinha, L. M. V., Gomes, F. S., \& Costa, A. W. N. (2020). Consumo e comportamento alimentar entre adolescentes brasileiros: Pesquisa Nacional de Saúde do Escolar (PeNSE), 2009. Ciencia \& Saúde Coletiva, 15(2), 3085-309.

Louzada, M. L. C., Ricardo, C. Z., Steele, E. M., Levy, R. B., Cannon G., \& Monteiro, C. A. (2018). The share of ultra-processed foods determines the overall nutritional quality of diets in Brazil. Public Health Nutr, 21(1), 94-102.

Milani, M. O., Garlet, L., Romero, G. G., \& Mattos, K. M. (2017). Influência da mídia nos hábitos alimentares de crianças: uma revisão da literatura. Rev. De epidemiologia e controle de infecção, 5(3), 01-05.

Nery, I. S., Mendonça, R. C. M., Gomes, I. S., Fernandes, A. C. N., \& Oliveira, D. C. (2017). Reincidência de gravidez em adolescentes de Teresina, PI, Brasil. Rev Bras Enferm.

Oliveira, R. Rodeghiero, Peter, N. B., \& Muniz, L. C. (2021). Consumo alimentar segundo grau de processamento entre adolescentes da zona rural de um município do sul do Brasil. Ciência \& Saúde Coletiva.

Pereira T. S., Pereira, R. C., Angelis-Pereira, M. C. (2017). Influência de intervenções educativas no conhecimento sobre alimentação e nutrição de adolescentes de uma escola pública. Ciência e saúde coletiva, 22(2).

Pinho, F. N. L. G., Martínez, S. A. (2014). História da merenda escolar no Brasil: Trajetórias da mais antiga política de suplementação alimentar brasileira (1955- 2012). Trabalho apresentado no II CONINTER - Congresso Internacional Interdisciplinar em Sociais e Humanidades, Belo Horizonte.

Pinto, L. P. (2017). Conhecimento dos pais sobre alimentação infantil: relação com as caraterísticas sociodemográficas e estado nutricional da criança. Repositório Científico do Instituto Politécnico de Viseu/ ESCOLA SUPERIOR DE SAÚDE DE VISEU/ Unidade de Enfermagem da Criança e do Adolescente (UECA).

Potter, P., \& Perry, A. G. (2013). Fundamentos de Enfermagem. Elsevier.

Reato, L.F.N. (2018). Mídia x Adolescente. Pediatria Moderna, 37, 37-40.

Santos, S.L. (2017). Influência da propaganda nos hábitos alimentares: análise de conteúdo de comerciais de alimentos da televisão. 2007.163 f. Dissertação (Mestrado em Engenharia de Produção) - Departamento de Engenharia de Produção, Universidade Federal de São Carlos, São Carlos.

Santos, L. A. S. O. (2019). Fazer educação alimentar e nutricional: algumas contribuições para reflexão. Ciência \& Saúde Coletiva, 17(2), p. 453-462.

Salvatti, A. G., Escrivão, M. A. M. S., Taddei, J. A. A. C., \& Bracco, M. M. (2017). Padrões Alimentares de adolescentes na cidade de São Paulo. Rev Nutr.

Silva, R.C.R, \& Malina, R.M. (2018). Nível de atividade física em adolescentes do Município de Niterói, Rio de Janeiro, Brasil. Cad. Saúde Pública, 16(4),1091-1097.

Silva, K. G. S., Magalhaes, M. J. S., Pessoa, N. M., Morais, E. R. O., Lima, A. S. S., Santos, M. E. L. D. C., \& Silva, L. A. S. (2021). Perfil alimentar de adolescentes no contexto contemporâneo: uma revisão integrativa. In: Laurinda Fernanda Saldanha Siqueira, Maynara Costa de Oliveira Silva. (Org.). Maternidade, aborto e direitos da mulher. 1ed.São Luís, MA: Editora Expressão Feminista, 1, 149-156.

Silva, K. G. S. D., Melo, K. C., Santos, M. E. L. D. C., Silva, A. T. D., Lima, A. S. S., Pessoa, N. M., \& Morais, T. O. (2021). Functional properties of babassu coconut mesocarp flour: a nutritional alternative against Covid-19. Research, Society and Development, 10(2).

Silva, K. G. S., Magalhaes, M. J. S., Borba, F. L., \& Sousa, M. R. S. (2019). Perfil alimentar de adolescentes na contemporaneidade: uma revisão. Revista Eletrônica Acervo em Saúde, 6, 48.

Soares, A. N., Melo, K. C., Silva, W. C. D., Silva, C. O. D., Silva, A. A. D., Silva, K. G. S. D., \& Sousa, F. D. C. A. (2021). Potenciais efeitos imunomoduladores de nutracêuticos no tratamento de covid-19. International Journal of Development Research, 11(4), 46233-46238.

Tavares, L. F., Yakoo, E. M., Rosa, M. L. G., \& Fonseca, S. C. (2017). Síndrome Metabólica em crianças e adolescentes brasileiros: Revisão sistemática. Caderno saúde coletiva.

Taddei, J.A., Lang, R. M. F., Longo-Silva, G., \& Toloni, M. H. A. (2021). Nutrição em Saúde Pública. Editora Rubio.

Toral, N., Conti, M. A., \& Slater, B. A alimentação saudável na ótica dos adolescentes: percepções e barreiras à sua implementação e características esperadas em materiais educativos. Cad. Saúde Pública. 25(11), 2386-2394, 2019.

Vieira, M. V., Del Ciampo, I. R. L., \& Del Ciampo, L. A. (2018). Hábitos e consumo alimentar entre adolescentes eutróficos e com excesso de peso. Rev Bras Crescimento Desenvolv Hum, 24(2), 157-162.

Veiga, G. V., Costa, R. S., Araujo, M. C, Souza, A. M., Bezerra, A. N., Barbosa, F. S., \& Pereira, R. A. (2018). Inadequação do consumo de nutrientes entre adolescentes brasileiros. Revista de Saúde Pública, 47(1), 212-221.

World Health Organization - WHO. (2014). Health for the world's adolescents. A second chance in the second decade. 